\title{
Periostin is a novel histological biomarker for the diagnosis of chondroid tumor
}

\author{
Dough Kim ${ }^{1,2}$, Ji Yun Jeong ${ }^{3,4}$, Man-Hoon Han ${ }^{3,4}$, Jongmin Chae ${ }^{3,4}$, Ilhyung Park ${ }^{5,6}$, Jongphil Yoon ${ }^{5,6}$, \\ Heesoo Kyung ${ }^{5,6}$, Ha-Jeong Kim ${ }^{1,2}$, Wonju Jeong ${ }^{5,6}$ \\ ${ }^{1}$ Department of Physiology, School of Medicine, Kyungpook National University, Daegu, South Korea; ${ }^{2}$ BK21 Plus KNU Biomedical Convergence \\ Program, Department of Biomedical Science, School of Medicine, Kyungpook National University, Daegu, South Korea; ${ }^{3}$ Department of Pathology, \\ School of Medicine, Kyungpook National University, Daegu, South Korea; ${ }^{4}$ Department of Pathology, Kyungpook National University Hospital, \\ Daegu, South Korea; ${ }^{5}$ Department of Orthopedic Surgery, School of Medicine, Kyungpook National University, Daegu, South Korea; ${ }^{6}$ Department \\ of Orthopedic Surgery, Kyungpook National University Hospital, Daegu, South Korea \\ Contributions: (I) Conception and design: HJ Kim, W Jeong; (II) Administrative support: D Kim; (III) Provision of study materials or patients: I Park, \\ J Yoon, H Kyung, W Jeong; (IV) Collection and assembly of data: JY Jeong, MH Han, J Chae, I Park, J Yoon, H Kyung, W Jeong; (V) Data analysis \\ and interpretation: D Kim, JY Jeong, MH Han, J Chae; (VI) Manuscript writing: All authors; (VII) Final approval of manuscript: All authors. \\ Correspondence to: Ha-Jeong Kim, MD, PhD. 680 Gukchaebosang-ro, Jung-gu, Daegu 41944, South Korea. Email. kimhajeong@knu.ac.kr; Wonju \\ Jeong, MD, PhD. 130 Dongdeok-ro, Jung-gu, Daegu 41944, South Korea. Email. wjjeong@knu.ac.kr.
}

Background: The chondroid tumor is generally classified into three types, enchondroma, low-grade chondrosarcoma, and high-grade chondrosarcoma. A histological evaluation of a biopsy sample is the best predictor of the clinical course in most patients with carcinomas or sarcomas. Sometimes serological or molecular markers are used as prediction markers, but there has been no reliable marker for chondroid tumor diagnosis. Clinical and radiological, but not histological features, are still used in the diagnosis and staging of chondroid tumors. During a histopathological diagnosis, it has been difficult to distinguish between benign enchondroma and low-grade chondrosarcoma. To allow for more accurate treatments, new histological biomarkers for the differential diagnosis are needed.

Methods: Twenty-eight cases of enchondromas and thirty-three cases of low-grade chondrosarcoma were selected. Thirteen cases of non-tumorous cartilage were used for the control group, who underwent artificial joint surgery for degenerative arthritis. Surgically removed tissue specimens were formalin-fixed paraffinembedded and hematoxylin and eosin (H\&E) and immunohistochemistry (IHC) stains were performed.

Results: Periostin was expressed in chondroid tumors but not in the normal cartilage. Periostin was observed via immunostaining in the cytoplasm but not in the extracellular matrix of enchondroma tissue, and was observed in the cytoplasm and extracellular matrix of low-grade chondrosarcoma. The sensitivity and specificity of these stains were $93.9 \%$ and $96.4 \%$, respectively.

Conclusions: Based on these results, we suggest that periostin could be used as a novel prognostic marker to distinguish between enchondroma and low-grade chondrosarcoma.

Keywords: Periostin; cartilage; enchondroma; low-grade chondrosarcoma; differential diagnosis marker

Submitted Jul 09, 2020. Accepted for publication Oct 30, 2020.

doi: $10.21037 /$ tcr-20-2499

View this article at: http://dx.doi.org/10.21037/tcr-20-2499

\section{Introduction}

Chondrosarcomas are cartilage-forming malignant tumors that affect adults between 30 and 60 years old. It is the second most common primary malignant type of bone malignancy, $10-12 \%$ of which are secondary bone malignancies while the rest are primary $(1,2)$. In the presence of previous lesions, they can be divided into primary and secondary chondrosarcomas. Furthermore, 
benign osteochondromas sometimes change to secondary chondrosarcoma.

Chondrosarcomas have shown a wide spectrum of clinical behaviors, and severe bone destruction and pathological fractures will be lea without the proper treatment. Wide surgical excision is the best treatment for intermediate- to high-grade chondrosarcomas. However, low grade chondrosarcoma can be treated with curettage and filling of the cavity. Advanced surgical techniques such as cryotherapy and additional treatments (including second surgery or additional chemotherapy) allows for better prognosis. Therefore, excessive dissection is performed to prevent metastasis and recurrence (3-5).

Chondrosarcomas are classified into several types by their location in the bone, the presence of previous lesions, and histological grades. Central and peripheral chondrosarcomas are divided by their location in bone including the intramedullary space or in the bony surfaces. Histopathologic grade is divided into grade 1,2, and 3 (low-, intermediate-, and high-grade) or low-grade (grade I) and high-grade (grade II) (6). Among these criteria, histopathological grade is the most significant prognostic factor in the chondroid tumor $(7,8)$. Even though histological classification is highly correlated with prognosis, inter-observer variation is difficult to control (2). The distinction between enchondromas (benign tumors) and grade I chondrosarcomas (malignant tumors) is extremely difficult based on classical hematoxylin and eosin (H\&E) staining as well as radiological and clinical information $(7,9,10)$. Therefore, the development of new biological markers to distinguish grades of cartilage tumors is essential.

Periostin was originally identified as an adhesion protein of the osteoblastic cell line of osteoblast specific factor-3 (OAF-3). It is a $93 \mathrm{kDa}$ protein composed of four fasciclin-1 (fas-1) domains which are known to be involved in cell adhesion. Periostin is mainly distributed in the periosteum and plays a physiological role in the induction and adherence of osteoblast precursors in the growth plate during endochondral bone formation (11). Recently, fibroblasts secreting periostin have been shown to be related to tumors in the growth and metastasis of lung, breast, colon, stomach, bladder, and head and neck cancers as well as melanomas $(12,13)$.

The purpose of this study was to evaluate periostin as a new biological marker by confirming the expression of periostin in chondrosarcoma tissue. We present the following article in accordance with the REMARK reporting checklist (available at: http://dx.doi.org/10.21037/ tcr-20-2499).

\section{Methods}

\section{Patient classification}

Patients were diagnosed with enchondromas or low-grade malignant chondrosarcomas through a clinicopathological conference composed of pathologists, radiologists, and orthopedic surgeons, and then were followed up for more than 2 years after surgery from March 2008 to February 2015. Twenty-eight cases of enchondromas and 33 cases of low-grade chondrosarcoma were selected. Thirteen cases of normal cartilage were used for the control group, who underwent artificial joint surgery for degenerative arthritis. The study was conducted in accordance with the Declaration of Helsinki (as revised in 2013 This study was approved by the IRB committee of Kyungpook National University Hospital (approval number 2015-10-004-001) and informed consent was taken from all the patients.

Age, sex, height, weight, and body mass index of patients with normal cartilage, enchondromas, and low-grade chondrosarcomas were not significantly different from one another except for age. As the normal cartilage tissue samples were harvested during arthroplasty for treatment of osteoarthritis, the average age of the patients with normal cartilage was 66.9 years (range, $56-75$ years). The mean ages of the patients with enchondromas and chondrosarcomas were 39.4 and 53.4 years (range, 17-63 and 23-72 years), respectively (Table 1). There were no statistically significant differences the group with the normal cartilage and chondroid tumors in the age, gender, height, weight, BMI, follow-up period, and treatment methods except for age. There were also no differences the group with the enchondromas and low-grade chondrosarcomas.

\section{H\&E and immunobistochemistry (IHC) stains}

The tumors were collected by surgical excision. Because of the heterogeneity of the sarcoma, it was necessary to reduce the selection bias when sampling the tumor. Therefore, all of the tumor tissues harvested from the surgery were embedded into paraffin blocks. All surgically removed tissue specimens were formalin-fixed and paraffin-embedded. To prepare the tissue slide, the decalcification step was performed before paraffin embedding. The tissue slide was obtained at a thickness of $4 \mu \mathrm{m}$. H\&E and IHC stains were performed by serial sectioning with the block that best 
Table 1 Demographic data on patients with normal cartilage, enchondroma and low grade chondrosarcoma

\begin{tabular}{|c|c|c|c|c|}
\hline Characteristics & Normal cartilage & Enchondroma & Low grade chondrosarcoma & $\mathrm{P}$ \\
\hline Age (year) & $66.9[56-75]$ & $39.4[17-63]$ & 53.4 [23-72] & $<0.01^{\mathrm{a}}$ \\
\hline $\operatorname{Sex}(M / F)$ & $4 / 9$ & $15 / 13$ & $13 / 20$ & $0.326^{b}$ \\
\hline Height $(\mathrm{cm})$ & $158.9[148-175]$ & 165.1 [141-183] & $164[150-182]$ & $0.073^{a}$ \\
\hline $\operatorname{BMI}\left(\mathrm{kg} / \mathrm{m}^{2}\right)$ & $24.4[20.9-28.8]$ & $22.6[16.8-31.4]$ & $22.8[16.0-30.8]$ & $0.228^{\mathrm{a}}$ \\
\hline
\end{tabular}

${ }^{\mathrm{a}}$, ANOVA test; ${ }^{\mathrm{b}}$, kai-square test.

represented the entire tumor.

$\mathrm{H} \& \mathrm{E}$ and IHC stains were performed using previously described protocols (14). Briefly, paraffin around the tissue was removed using xylene, and hydration was performed for $10 \mathrm{~min}$ at $90 \%, 80 \%$, and $70 \%$, respectively, of the gradually decreasing ethanol solution. Tissues were placed in $0.01 \mathrm{M}$ citric acid for antigen retrieval. Endogenous peroxidase and nonspecific antigens were blocked with 3\% hydrogen peroxide solution and 5\% normal goat serum. IHC staining was performed using a rabbit anti-human periostin monoclonal antibody (Abcam, CA) and goat antirabbit IgG-HRP (Santa Cruz Biotechnology Inc., CA, USA). Each step was washed with phosphate buffered saline (PBS). The final colorimetric reaction was performed with diaminobenzidine (DAB).

\section{Pathology analysis}

Three pathologists separately classified the nucleus, cytoplasm, and extracellular matrix of cartilaginous tissue or chondroid tumors of all tissue slides as strong positive $(++)$, positive $(+)$, weak positive $(+/-)$, or negative $(-)$. The intensity as well as the positive and negative criteria of the IHC stain were determined based on the consultation and meeting of the three pathologists. The criteria for negative stains include contaminations, non-selective stains with antibody, stains of extra-cartilaginous tissues, and less than $5 \%$ of the stain (Figure S1).

The serial section identified as cancer tissue by $\mathrm{H} \& \mathrm{E}$ staining was used for IHC staining. Clinical and radiologic results were also provided.

\section{Statistical analysis}

Statistical analysis was performed using independent $t$-test, ANOVA test, Chi-square test and Fisher exact test using SPSS version 17.0 (SPSS Inc., Chicago, IL,USA). The specificity and sensitivity of the test were analyzed. Mathematically, sensitivity is calculated the percentage that diagnostic test is positive for the disease, given that the subject actually has the disease. Specificity is calculated the percentage that the diagnostic test is negative, given that the subject does not have the disease.

\section{Results}

\section{Periostin expression in the chondroid tumor tissues and the normal cartilages}

Cartilage deformity was diagnosed by a simple weight bearing radiogram (Figure 1A), and normal cartilage tissue without a chondroid tumor was confirmed at the operation site (Figure $1 B$ ) during the artificial joint surgery. $\mathrm{H} \& \mathrm{E}$ stained images showed that the collected cartilage tissue was normal (Figure 1C,D,E). Periostin was not observed in the normal cartilage (Figure $1 F, G, H$ ).

A 22-year-old woman was suspected of having an enchondroma because of a developing chondroid tumor in the small bone, the first proximal phalanx, as indicated by a simple radiogram (Figure $2 A$ ), computed tomography (CT) imaging (Figure 2B), and magnetic resonance imaging (MRI) (Figure 2C). The tumor was surgically removed, and the tissues were analyzed. As shown in Figure 2, the removed tissue was confirmed to be enchondroma by H\&E staining. Periostin expression was observed in the cytoplasm of the enchondroma cells but not in the nuclei and extracellular matrix.

A 45-year-old woman had a tumor in her right humerus. The tumor was suspected as low-grade chondrosarcoma because of a developing chondroid tumor in the long bone, 
A

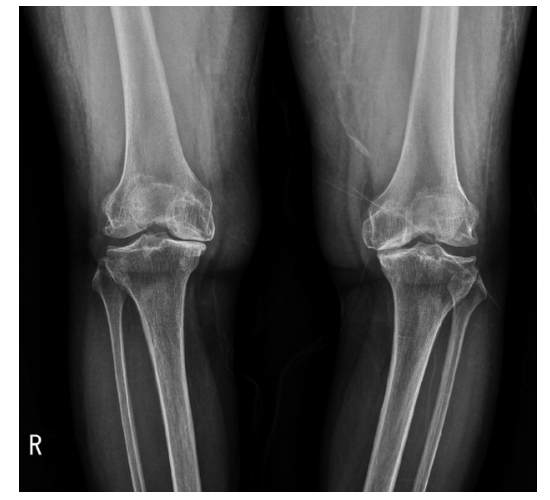

C
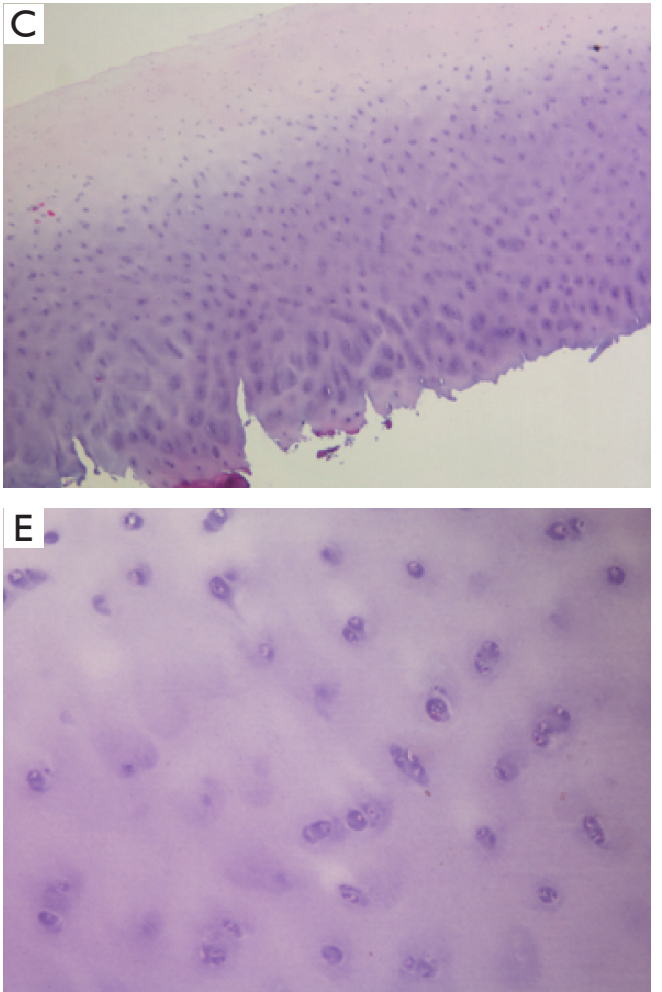

G

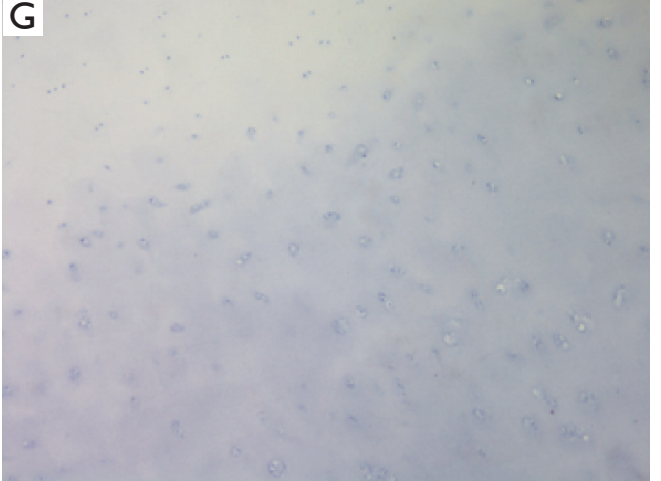

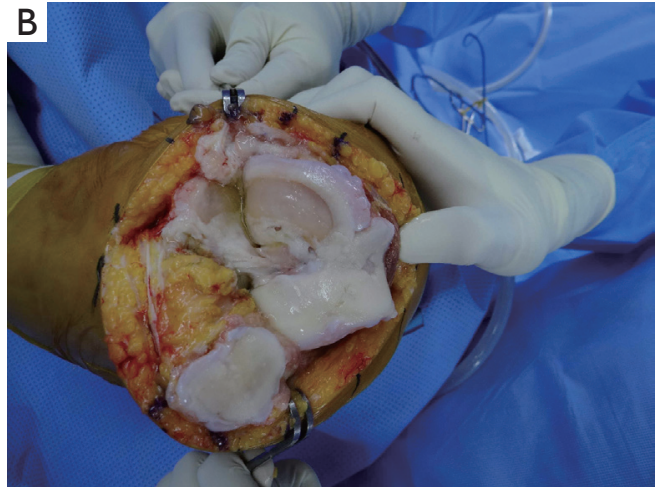
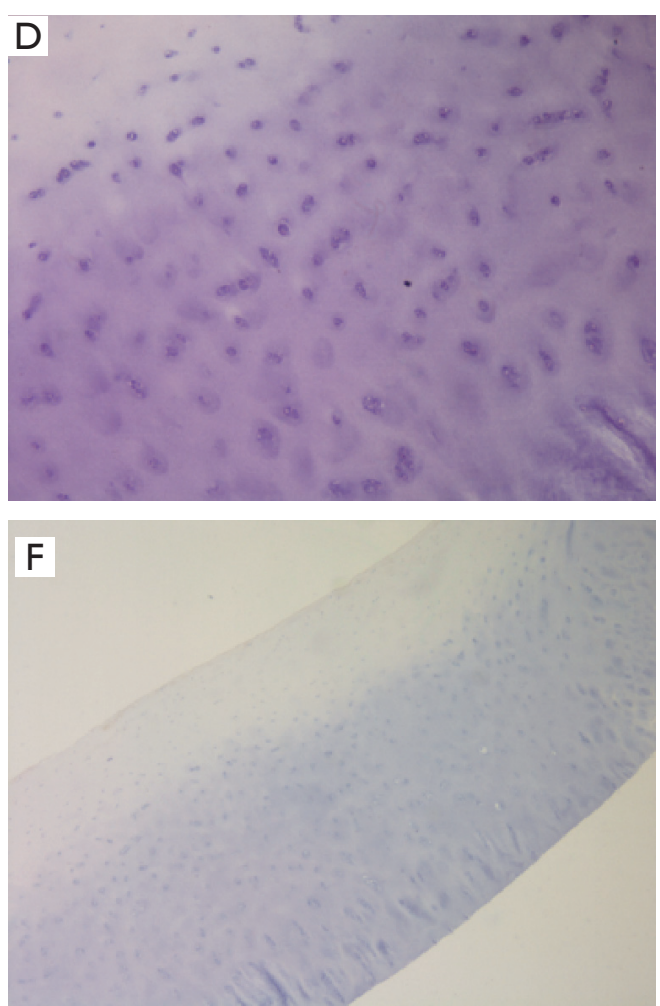

$\mathrm{H}$

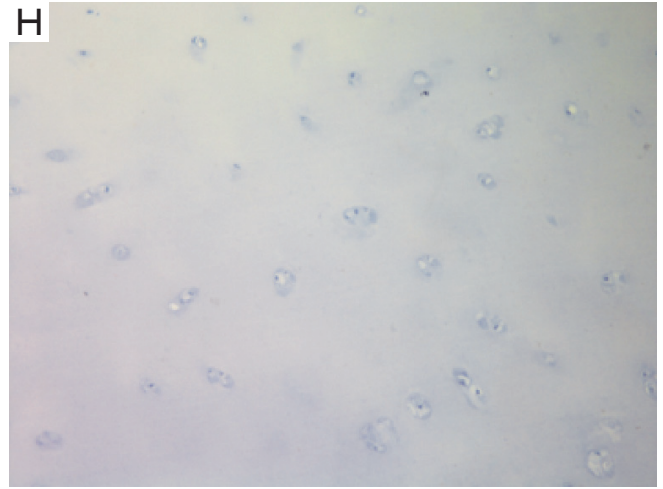

Figure 1 Normal cartilage. A 68-year-old female had the degenerative arthritis in left knee joint. Both knee simple radiogram of both knee (A). Intraoperative optical cartilage image (B). H\&E stain image (C,D,E). Immunohistochemistry stain of periostin (F,G,H). Images were taken under 10× (C,F), 20× (D,G), and 400× (E,H) magnification field. 
A
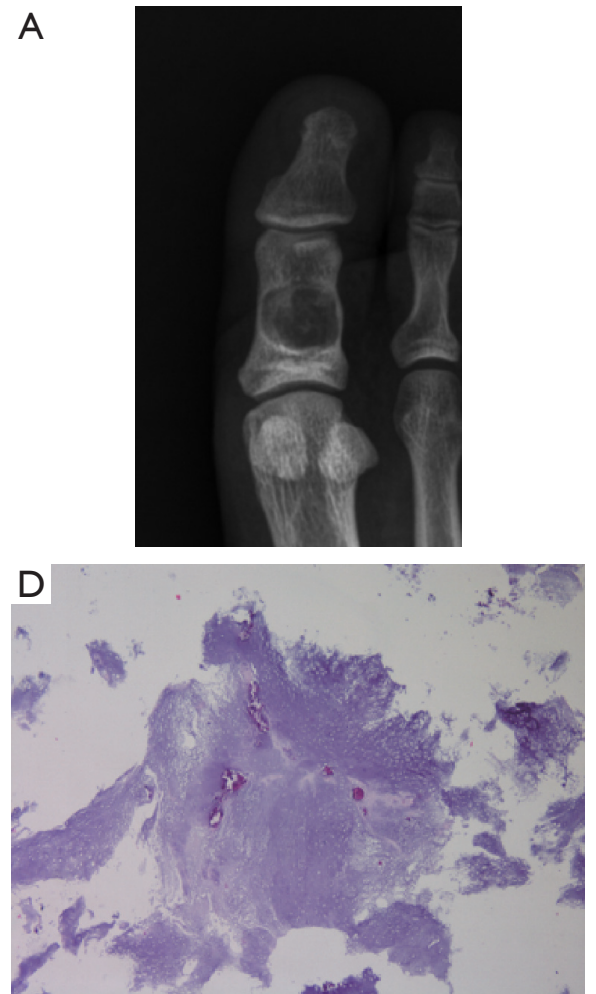

G

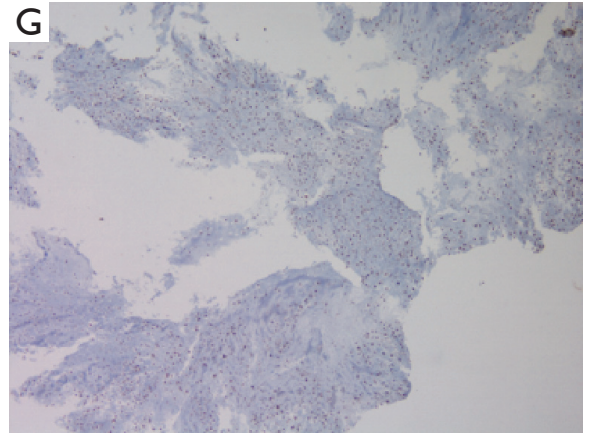

B

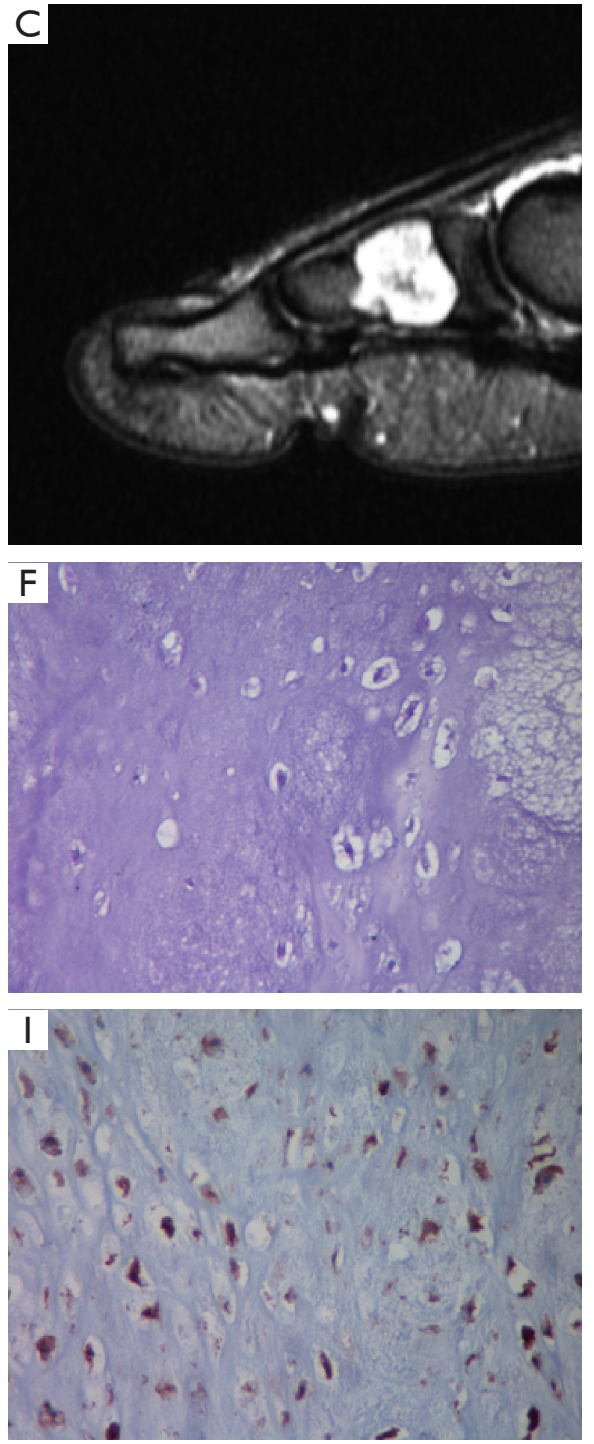

Figure 2 Enchondroma. A 22-year-old woman had the enchondroma in her first proximal phalanx. Simple radiogram (A). CT image (B). MRI image (C). H\&E stain image (D,E,F). Immunohistochemistry stain of periostin (G,H,I). Images were taken under 10× (D and G), 20× $(\mathrm{E}, \mathrm{H})$, and $400 \times(\mathrm{F}, \mathrm{I})$ magnification field.

the right humerus, by a simple radiogram (Figure 3A), CT imaging (Figure 3B), and MRI imaging (Figure 3C), and it was thus surgically excised. After H\&E staining, it was diagnosed as low-grade chondrosarcoma (Figure 3D,E,F). Periostin was expressed in the nuclei, cytoplasm, and extracellular matrix (Figure 3G,H,I).

These three results suggest that periostin was not expressed in normal chondrocytes, but it increased when chondrocytes progressed to oncogenesis.

\section{Periostin expression patterns analysis}

Thirteen cases of normal cartilage, 28 cases of enchondroma, and 33 cases of low-grade chondrosarcoma tissues were used. Periostin was not observed in any of 13 cases of normal cartilages. In all 28 cases of enchondromas, periostin was observed in the cytoplasm, but not in the nucleus and the extracellular matrix. In only one enchondroma case, periostin was observed in the cytoplasm and the extracellular matrix. Periostin was observed in 32 cases 

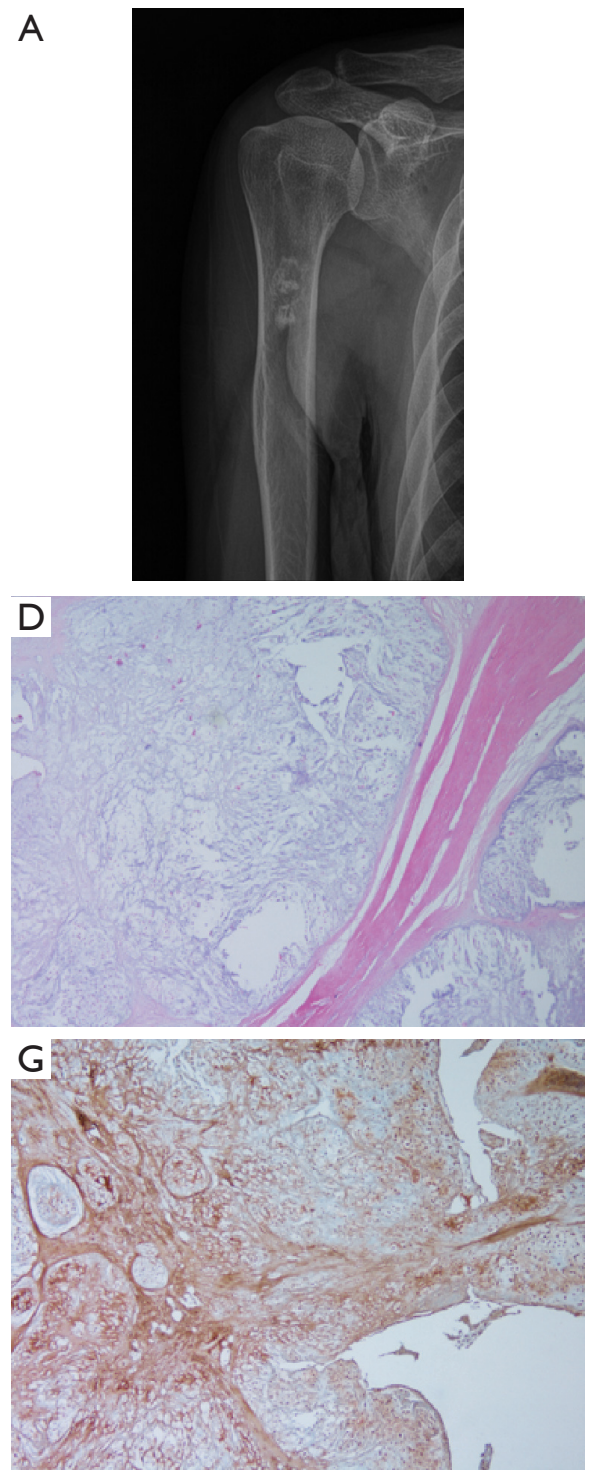
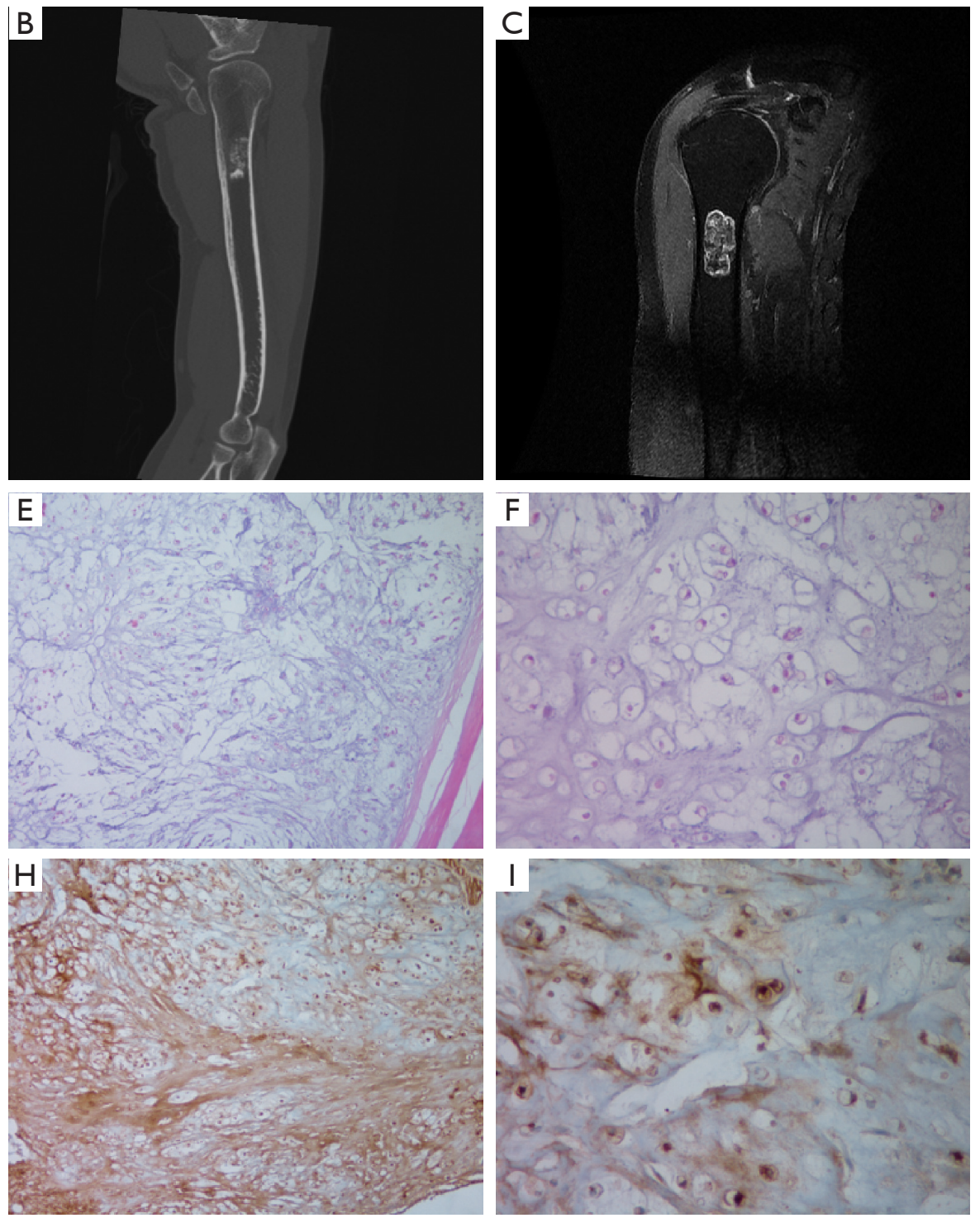

Figure 3 Low-grade chondrosarcoma. A 45-year-old woman had the low-grade chondrosarcoma in right humerus. Simple radiogram of right humerus (A). CT image (B). MRI image (C). H\&E stain image (D,E,F). Immunohistochemistry stain of periostin (G,H,I). Images were taken under $10 \times(\mathrm{D}, \mathrm{G}), 20 \times(\mathrm{E}, \mathrm{H})$, and $400 \times(\mathrm{F}, \mathrm{I})$ magnification field.

of low-grade chondrosarcoma in the nuclei, cytoplasm, and/or extracellular matrix. In one case of low-grade chondrosarcoma, periostin was not observed anywhere (Table 2). The sensitivity and specificity for the diagnosis were $98.4 \%$ and $100 \%$ (Fisher exact test, $\mathrm{P}<0.01$ ), respectively, when the normal cartilaginous tissue and chondroid tumors were classified as positive and negative by periostin stain (Table 2).

Periostin was not observed in the nucleus of chondrocytes in enchondromas but was observed in some cases (4/33) of low-grade chondrosarcomas (Table 2). The sensitivity and specificity for differential diagnosis were $12.1 \%$ and $100 \%$ (Fisher exact test, $\mathrm{P}=0.12$ ), respectively, when the enchondromas and low-grade chondrosarcomas were classified as positive and negative by the periostin stain in the nucleus.

Periostin was present in the cytoplasm of all chondroid tumor tissue samples except for one case of low-grade 
Table 2 Periostin expression in the nucleus $(\mathrm{N})$, cytoplasm $(\mathrm{C})$, or extra cellular matrix (ECM)

\begin{tabular}{|c|c|c|c|c|c|c|c|c|c|}
\hline Periostin stain & \multicolumn{3}{|c|}{ Normal cartilages } & \multicolumn{3}{|c|}{ Enchondroma } & \multicolumn{3}{|c|}{ Low grade chondrosarcoma } \\
\hline$(-)$ & 13 & 13 & 13 & 28 & 0 & 27 & 29 & 1 & 15 \\
\hline$(+)$ & 0 & 0 & 0 & 0 & 28 & 1 & 4 & 32 & 18 \\
\hline Total & & 13 & & & 28 & & & 33 & \\
\hline
\end{tabular}

chondrosarcoma (Table 2). The sensitivity and specificity for the differential diagnosis were $96.9 \%$ and $0 \%$ (Fisher exact test, $\mathrm{P}=0.27$ ), respectively, when the enchondroma and low grade chondrosarcoma were classified as positive and negative by the periostin stain in the cytoplasm.

The degree of the periostin stain in the cytoplasm of the chondrocytes was different between the enchondroma and low-grade chondrosarcoma. The cytoplasmic periostin expression in low-grade chondrosarcomas was weaker than that in enchondromas. The cytoplasm of the chondrocytes was strongly stained with periostin in all of the enchondroma, and the extracellular matrix was not stained except one case (Figure $4 A, B$ ). In $62.5 \%$ of lowgrade chondrosarcomas, periostin was weakly stained in the cytoplasm (Table 3). When the cytoplasm was strongly stained with periostin in $37.5 \%$ of the low-grade chondrosarcoma, the extracellular matrix was also stained with periostin except two cases (Figure 4C,D,E,F). Periostin was not observed in the extracellular matrix of all of the case of enchondroma except for one, whereas it was positive in $54.5 \%$ of the cases of low-grade chondrosarcoma (Table 2).

\section{Discussion}

The differential diagnosis between enchondromas and low-grade chondrosarcomas is necessary to determine the best treatment. Conserved surgical excision is sufficient to improve the patients quality of life in those with enchondromas because enchondromas are benign and rarely metastasized. However, aggressive extensive cuttage is performed in some patients diagnosed with enchondromas but clinically suspected of low-grade chondrosarcomas to reduce the possibility of metastasis and recurrence because the differential diagnosis between enchondroma and lowgrade chondrosarcoma is difficult by histologic analysis $(10,15)$. Biomarkers for differential diagnoses are urgently needed, but so far there has been no accurate markers. Here, we suggest periostin as a new diagnostic marker to distinguish between enchondromas and low-grade chondrosarcomas.

In this study, 13 normal cartilage, 28 enchondroma, and 33 low-grade chondrosarcoma tissue samples were used and analyzed its periostin staining pattern and staining intensity degree in the chondroid tissues (Table 2). Periostin is known as a stromal associated protein, but it is not found in normal cartilage tissue (Figure 1). Periostin was only found in chondroid tumors (Figures 2,3), except in one case of low-grade chondrosarcoma (Table 2). The sensitivity and specificity were $98.4 \%$ and $100 \%$ (Fisher exact test, $\mathrm{P}<0.01)$ in the diagnosis of chondroid tumors when periostin expression was used as a marker. When the staining intensity of the cytoplasm and the presence or absence of extracellular matrix staining was used, the sensitivity and specificity are $93.9 \%$ and $96.4 \%$ (Fisher exact test, $\mathrm{P}<0.01$ ), respectively, in the differential diagnosis between enchondromas and low-grade chondrosarcomas (Table 4). This result suggests that periostin can be used as a biomarker for the differential diagnosis between enchondromas and low-grade chondrosarcomas. Another previous study also reported that periostin was observed in low-grade chondrosarcomas (14). In that study, the authors identified periostin by a LC-MS/MS approach in formalin-fixed, paraffin-embedded tissue samples, and observed periostin in 31 enchondroma and 23 low-grade chondrosarcoma tissue samples, and observed periostin in extracellular matrix of chondrosarcomas but not in enchondromas (14). Their findings in the enchondromas differed from our findings. This may be due to limitations in immunohistochemistry staining and the retrospective studies. More long-term follow-up studies of more cases by other groups are needed to confirm periostin as a biomarker.

Periostin was overexpressed in various types of human carcinomas $(12,13)$. In carcinoma tissues, it is secreted from cancer-associated fibroblasts, and supports cancer stem cell formation, angiogenesis, and lymphangiogenesis (16). 

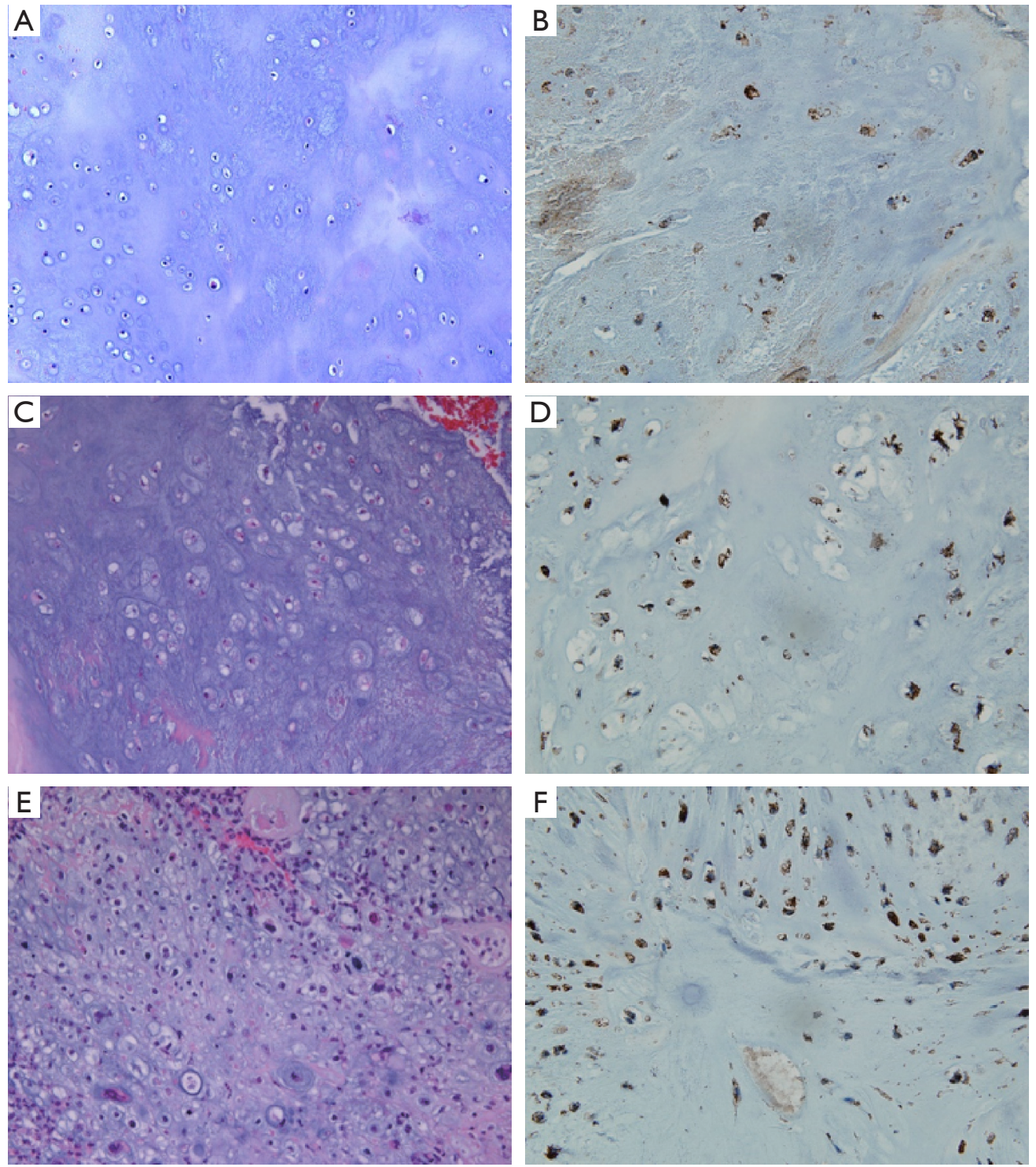

Figure 4 Periostin stain in cytoplasm of enchondroma and low-grade chondrosarcoma tissue. H\&E stain images (A,C,E) were taken under $200 \times$ magnification field. Immunohistochemistry stain of periostin images (B,D,F) were taken under 400× magnification field.

It was also reported that periostin was significantly correlated with angiogenesis and poor prognosis in osteosarcomas (17). Periostin in osteosarcoma may be secreted from dedifferentiated osteosarcoma cells because it was identified from the MC3T3-E1 osteoblastic cell line (18). From these findings, periostin in chondrosarcomas may be secreted from chondrosarcoma cells and/or tumorassociated mesenchymal stem cells, and it may enhance the stemness of chondroid cancer cells, angiogenesis, and lymphangiogenesis in sarcomas, similar to its role in carcinomas. Furthermore, the periostin expression pattern in chondroid tissue may provide on clues on its role in chondroid oncogenesis.

The periostin expression pattern in chondroid tumor tissue was obviously different in benign enchondromas and malignant low-grade chondrosarcomas. In enchondroma tissue, periostin was only observed in the cytoplasm but not in the nuclei and extracellular matrix. Periostin expression in the extracellular matrix was only found in low-grade chondrosarcomas (Figures 2,3, and Table 2). This can be 
Table 3 The degree of periostin stain in the cytoplasm between enchondroma and low-grade chondrosarcoma

\begin{tabular}{lcc}
\hline Periostin stain & Enchondroma & $\begin{array}{c}\text { Low grade } \\
\text { chondrosarcoma }\end{array}$ \\
\hline Cytoplasm & 28 & 12 \\
$\begin{array}{l}\text { Strong positive (++) } \\
\text { Positive (+), and weak }\end{array}$ & 0 & 20 \\
positive (+/-) & 28 & $32^{\mathrm{e}}$ \\
Total & $28 \%$, P<0.01 Fisher exact test.
\end{tabular}

Sensitivity: $62.5 \%$, specificity: $100 \%, \mathrm{P}<0.01$ Fisher exact test.

, exclusion; low grade chondrosarcoma (1 case) which the periostin stain of nucleus, cytoplasm, and extracellular matrix (ECM) was negative.

Table 4 The degree of periostin stain of cytoplasm and extracellular matrix (ECM) between enchondroma and low-grade chondrosarcoma

\begin{tabular}{lcc}
\hline Periostin stain & Enchondroma & $\begin{array}{c}\text { Low grade } \\
\text { chondrosarcoma }\end{array}$ \\
\hline $\begin{array}{l}\text { Cytoplasm: strong positive } \\
(++) \text { and ECM: negative (-) }\end{array}$ & 27 & 2 \\
$\begin{array}{l}\text { Cytoplasm: positive (+), weak } \\
\text { positive (+/-) or ECM: positive }\end{array}$ & 1 & 31 \\
Total & 28 & 33 \\
\hline
\end{tabular}

Sensitivity: 93.9\%, specificity: $96.4 \%, \mathrm{P}<0.01$ Fisher exact test.

explained by the degree of dysplasia of chondrocytes. Undefined oncogenes or tumor suppressor genes trigger periostin expression during chondroid tissue dysplasia, and periostin is secreted as malignancy progresses. It is also possible that periostin is secreted from other stromal cells in chondrosarcomas to trigger malignancy. Further studies on the role of periostin in chondrosarcomas will help elucidate its role in the process of sarcoma oncogenesis. In only one case of enchondroma, periostin was observed in the extracellular matrix (Figure $4 A, B$ ), similar to that of low-grade chondrosarcomas. That patient's tumor was suspected to be an enchondroma and therefore she received conservative operation. If her tumor was low grade chondrosarcoma instead and not enchondroma, the chance of recurrence and metastasis would have been increased. Closed observations and long follow-up are needed to determine recurrence and metastasis.

The periostin expression pattern was more heterogeneous in low-grade chondrosarcomas than in enchondromas (Table 2). In 4 cases of low-grade chondrosarcoma, periostin was observed in the nucleus, cytoplasm, and extracellular matrix (Figure 3). Based on periostin nuclear expression, the sensitivity and specificity was $12.1 \%$ and $100 \%$ (Fisher exact test, $\mathrm{P}=0.12$ ), respectively. Periostin was found in the extracellular matrix in 18 cases of low-grade chondrosarcoma but not in enchondromas (Table 2). The sensitivity and specificity were $54.4 \%$ and $96.4 \%$ (Fisher exact test, $\mathrm{P}<0.01$ ), respectively, based on the periostin stain of the extracellular matrix of the chondroid tumor. When the extracellular matrix in the low-grade chondrosarcoma was stained for periostin, the degree of the cytoplasmic stain was weaker than that observed in enchondromas (Figures 2,3, and Table 3). In 12 cases of the low-grade chondrosarcomas, periostin was strongly observed in the cytoplasm, similar to that of enchondromas, but the extracellular matrix was also stained contrast to that observed in enchondromas (Figures 2,3, and Table 4). However, by the cytoplasmic staining intensity, the sensitivity and specificity were $100 \%$ and $62.5 \%$ (Fisher exact test, $\mathrm{P}<0.01$ ), respectively, when the malignant cartilage tumor was diagnosed as a malignant cartilage tumor (Table 3). In two cases of lowgrade chondrosarcomas, periostin was stained strongly in the cytoplasm and not in the extracellular matrix, such as that observed in enchondromas (Figure $4 C, D, E, F$ ). If it was enchondroma, the wide surgical excision that they had, was the overtreatment.

\section{Conclusions}

In this study, we revealed a marked difference in staining between normal cartilage, enchondroma, and lowgrade chondrosarcomas in the nucleus, cytoplasm, and extracellular matrix. The normal cartilage samples had no stains, whereas the enchondromas had strong staining in the cytoplasm not extracellular matrix, and low-grade chondrosarcomas had staining in the cytoplasm and extracellular matrix. Based on these results, the sensitivity and specificity were $97.0 \%$ and $96.4 \%$, respectively. This result means periostin may be useful for the differential diagnosis of enchondromas and low-grade chondrosarcomas.

\section{Acknowledgments}

Funding: This work was supported by Biomedical Research Institute grant, Kyungpook National University Hospital [2015]. 


\section{Footnote}

Reporting Checklist: The authors have completed the REMARK reporting checklist. Available at http://dx.doi. org/10.21037/tcr-20-2499

Data Sharing Statement: Available at http://dx.doi. org/10.21037/tcr-20-2499

Conflicts of Interest: All authors have completed the ICMJE uniform disclosure form (available at http://dx.doi. org/10.21037/tcr-20-2499). The authors have no conflicts of interest to declare.

Ethical Statement: The authors are accountable for all aspects of the work in ensuring that questions related to the accuracy or integrity of any part of the work are appropriately investigated and resolved. The study was conducted in accordance with the Declaration of Helsinki (as revised in 2013). This study was approved by the IRB committee of Kyungpook National University Hospital (approval number 2015-10-004-001) and informed consent was taken from all the patients.

Open Access Statement: This is an Open Access article distributed in accordance with the Creative Commons Attribution-NonCommercial-NoDerivs 4.0 International License (CC BY-NC-ND 4.0), which permits the noncommercial replication and distribution of the article with the strict proviso that no changes or edits are made and the original work is properly cited (including links to both the formal publication through the relevant DOI and the license). See: https://creativecommons.org/licenses/by-nc-nd/4.0/.

\section{References}

1. Hogendoorn PC, ESMO/EUROBONET Working Group, Athanasou N, et al. Bone sarcomas: ESMO Clinical Practice Guidelines for diagnosis, treatment and follow-up. Ann Oncol 2010;21 Suppl 5:v204-13.

2. Gelderblom H, Hogendoorn PC, Dijkstra SD, et al. The clinical approach towards chondrosarcoma. Oncologist 2008;13:320-9.

3. Dahlin DC, Henderson ED. Chondrosarcoma, a surgical and pathological problem; review of 212 cases. J Bone Joint Surg Am 1956;38-A:1025-38; passim.

4. Mohler DG, Chiu R, McCall DA, et al. Curettage and cryosurgery for low-grade cartilage tumors is associated with low recurrence and high function. Clin Orthop Relat Res 2010;468:2765-73.

5. Sambri A, Rocca M, Tuzzato G, et al. Lung Metastasectomy Improves Survival in Patients Affected by Grade 2 and 3 Chondrosarcoma. Oncol Res Treat 2020;43:134-9.

6. Ahlmann ER, Menendez LR, Fedenko AN, et al. Influence of cryosurgery on treatment outcome of low-grade chondrosarcoma. Clin Orthop Relat Res 2006;451:201-7.

7. Weiner SD. Enchondroma and chondrosarcoma of bone: clinical, radiologic, and histologic differentiation. Instr Course Lect 2004;53:645-9.

8. Sambri A, Tuzzato G, Donati DM, et al. Pathological fracture does not affect prognosis in dedifferentiated chondrosarcoma of the limbs. J Orthop Sci 2020. doi: 10.1016/j.jos.2020.04.013.

9. Ferrer-Santacreu EM, Ortiz-Cruz EJ, GonzálezLópez JM, et al. Enchondroma versus Low-Grade Chondrosarcoma in Appendicular Skeleton: Clinical and Radiological Criteria. J Oncol 2012;2012:437958.

10. Jeong W, Kim HJ. Biomarkers of chondrosarcoma. J Clin Pathol 2018;71:579-83.

11. Horiuchi K, Amizuka N, Takeshita S, et al. Identification and characterization of a novel protein, periostin, with restricted expression to periosteum and periodontal ligament and increased expression by transforming growth factor beta. J Bone Miner Res 1999;14:1239-49.

12. Ishiba T, Nagahara $M$, Nakagawa $T$, et al. Periostin suppression induces decorin secretion leading to reduced breast cancer cell motility and invasion. Sci Rep 2014;4:7069.

13. Zhu M, Fejzo MS, Anderson L, et al. Periostin promotes ovarian cancer angiogenesis and metastasis. Gynecol Oncol 2010;119:337-44.

14. Lai X, Chen S. Identification of novel biomarker candidates for immunohistochemical diagnosis to distinguish low-grade chondrosarcoma from enchondroma. Proteomics 2015;15:2358-68.

15. Mirra JM, Gold R, Downs J, et al. A new histologic approach to the differentiation of enchondroma and chondrosarcoma of the bones. A clinicopathologic analysis of 51 cases. Clin Orthop Relat Res 1985;(201):214-37.

16. Mosher DF, Johansson MW, Gillis ME, et al. Periostin and TGF- $\beta$-induced protein: Two peas in a pod? Crit Rev Biochem Mol Biol 2015;50:427-39.

17. Hu F, Shang XF, Wang W, et al. High-level expression of periostin is significantly correlated with tumour 
angiogenesis and poor prognosis in osteosarcoma. Int J Exp Pathol 2016;97:86-92.

18. Takeshita S, Kikuno R, Tezuka K, et al. Osteoblast-specific factor 2: cloning of a putative bone adhesion protein with homology with the insect protein fasciclin I. Biochem J 1993;294:271-8.

Cite this article as: Kim D, Jeong JY, Han MH, Chae J, Park I, Yoon J, Kyung H, Kim HJ, Jeong W. Periostin is a novel histological biomarker for the diagnosis of chondroid tumor. Transl Cancer Res 2021;10(1):434-444. doi: 10.21037/tcr-20-2499 

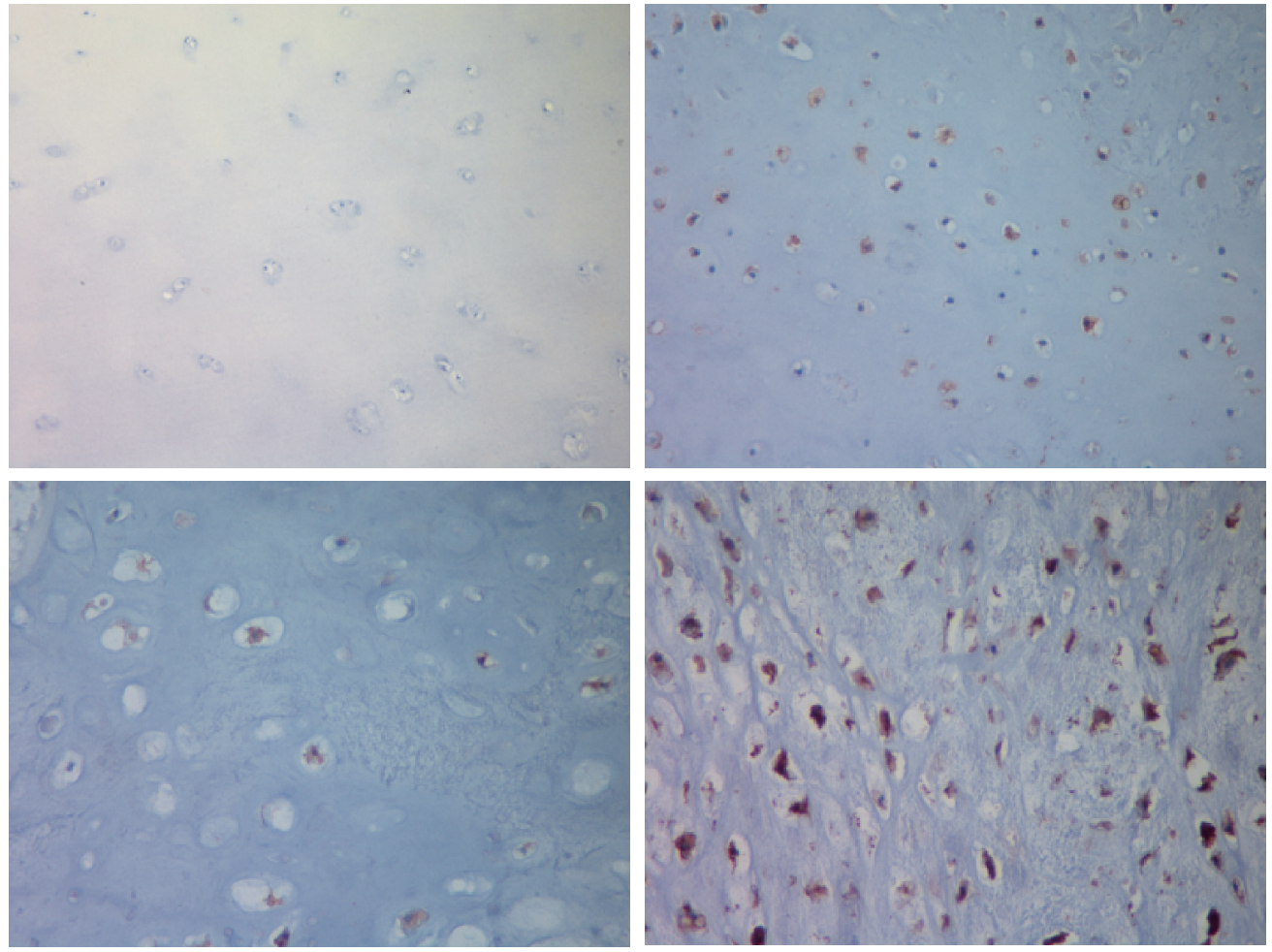

Figure S1 The criteria of IHC staining intensity. The cytoplasm of cartilaginous tissue or chondroid tumors are stained as negative (-), weak positive (+/-), positive (+) or strong positive (++). All images were taken under 400× magnification field. 